\title{
Connecting Two Stochastic Theories That Lead to Quantum Mechanics
}

\author{
Luis de la Peña*, Ana María Cetto and Andrea Valdés-Hernández \\ Departamento de Física Teórica, Instituto de Física, Universidad Nacional Autónoma de México, Mexico City, Mexico
}

The connection is established between two theories that have developed independently with the aim to describe quantum mechanics as a stochastic process, namely stochastic quantum mechanics (SQM) and stochastic electrodynamics (SED). Important commonalities and complementarities between the two theories are identified, notwithstanding their dissimilar origins and approaches. Further, the dynamical equation of SQM is completed with the radiation terms that are an integral element in SED. The central problem of the transition to the quantum dynamics is addressed, pointing to the key role of diffusion in the emergence of quantization.

Keywords: stochastic theories, foundations of quantum mechanics, stochastic electrodynamics (SED), stochastic mechanics, quantum fluctuations

\section{INTRODUCTION}

A whole and diverse series of stochastic theories have been developed with the aim to throw

OPEN ACCESS

Edited by:

Manuel Asorey,

University of Zaragoza, Spain

Reviewed by:

Gastao Inacio Krein,

São Paulo State University, Brazil

Andrei Khrennikov,

Linnaeus University, Sweden

*Correspondence:

Luis de la Peña

luis@fisica.unam.mx

Specialty section:

This article was submitted to

Mathematical Physics,

a section of the journal

Frontiers in Physics

Received: 12 February 2020 Accepted: 20 April 2020 Published: 12 May 2020

Citation:

de la Peña L, Cetto AM and Valdés-Hernández A (2020) Connecting Two Stochastic Theories That Lead to Quantum Mechanics. Front. Phys. 8:162.

doi: 10.3389/fphy.2020.00162 some light on the nature of the quantum phenomenon [for some representative work see Fényes [1-16]]. In this paper we pay attention to two theories in particular that have developed separately with the common purpose of describing the quantum phenomenon as a stochastic process. On one hand we have stochastic quantum mechanics, SQM (also known as stochastic mechanics), a phenomenological theory initiated by E. Nelson, and further developed and extended independently by several groups; a sample of related works is provided in Nelson [2], de la Peña [3], Guerra [4], Gaveau et al. [5], Nelson [6], de la Peña and Cetto [7], and Nelson [8], and references therein. On the other hand we have stochastic electrodynamics, SED, a first-principles theory pioneered by Marshall [11, 12] and Boyer [13] and further developed and completed with the contributions from a number of other authors, as shown in de la Peña and Cetto [7, 16], de la Peña et al. [17] Claverie [14], and Santos [15], and references contained therein. A common feature of these two theories is the explicit introduction of stochasticity as an ontological element missing in the quantum theory, with the aim to address many of the historical—and still current-conceptual difficulties associated with quantum mechanics. It is in a way astounding that the two theories have lived parallel lives for decades, virtually in isolation from one another.

In both SQM and SED the dynamics of a representative particle of mass $m$ is considered, for simplicity. In the phenomenological approach of SQM the (statistical) concepts of a flux velocity $v$ and a diffusive velocity $\boldsymbol{u}$ are introduced on an equal footing, without the need to specify the source of stochasticity. A generic equation of motion is obtained, which serves to describe the dynamics of two distinct types of stochastic process, in the Markov approximation: the classical, Brownianmotion type and the quantum one. The mathematics are simple and straightforward, and their physical meaning is clear.

The approach of SED, on the other hand, is guided by the hypothesis of the existence of the (random) zero-point radiation field, $\mathrm{ZPF}^{1}$. This rather more elaborate approach goes through a

\footnotetext{
${ }^{1}$ In the atomic, non-relativistic case it is sufficient to consider the electromagnetic vacuum; for other particles different kinds of vacua may have to be considered. A general formulation embracing all kinds of particles could be envisaged, based on a fluctuating spacetime; the different vacuum fields would then be manifestations of these primordial fluctuations.
} 
statistical evolution equation (a generalized Fokker-Planck-type equation, GFPE) in phase space, to arrive at a description in $\boldsymbol{x}$-space, in which the dissipative and diffusive terms are seen to bring about a definitive departure from the classical Hamiltonian dynamics. The interplay between these two terms is what allows the system to eventually reach equilibrium and thus attain the quantum regime; the dynamics is then described by the Schrödinger equation, and the operators become a natural tool for its description. Planck's constant enters into the picture through the spectral density of the ZPF, and this allows to determine uniquely the value of the only free parameter introduced in SED, as well as in SQM.

The purpose of the present work is to establish the connection between SQM and SED and, by so doing, to identify the strengths and limitations of the two theories, as well as certain commonalities and complementarities between them. With this aim, we first present the basic elements of SQM leading to the dynamical law that governs both classical and quantum stochastic processes in the Markov approximation. Secondly, we briefly review the statistical treatment followed in SED to arrive at a description in configuration space, and discuss the conditions under which the system attains equilibrium and thus reaches the quantum regime as described by the Schrödinger equation, which corresponds to the radiationless approximation of SED. The discussion of the connections between the two theories provides an opportunity to highlight the role played by diffusion in quantum mechanics. The more complete dynamical description provided by SED, which includes the radiative terms, serves in its turn to complete the corresponding dynamical equation of SQM. The distinct nature of the diffusive terms allows us to address the central problem of the transition from the initially classical dynamics with ZPF, to the quantum one. It is concluded that this more complete ontology which includes the ZPF as the source of stochasticity, leads in a natural process to the quantum description.

\section{THE UNDERLYING EQUATIONS OF STOCHASTIC QUANTUM MECHANICS}

Stochastic quantum mechanics is a phenomenological theory that considers a particle of mass $m$ undergoing a stochastic motion. It is general enough as to accommodate a range of physical phenomena in which an underlying stochastic process, considered in the Markov (second-order) approximation, takes place. The stochastic nature of the dynamics calls for a statistical treatment, which is carried out in $\boldsymbol{x}$-space. The basic kinematic elements for the description are obtained by applying an average over the ensemble of particles in the neighborhood of $\boldsymbol{x}$ at times close to $t$. By taking the time interval $\Delta t$ small but different from zero, two different velocities are obtained, namely the flux (or systematic) velocity [see e.g., [2, 7]]

$$
v(x, t)=\frac{\overline{x(t+\Delta t)-x(t-\Delta t)}}{2 \Delta t}=\hat{\mathcal{D}}_{c} x,
$$

with

$$
\hat{\mathcal{D}}_{c}=\frac{\partial}{\partial t}+\boldsymbol{v} \cdot \nabla
$$

and the diffusive (or osmotic) velocity

$$
\boldsymbol{u}(\boldsymbol{x}, t)=\frac{\overline{\boldsymbol{x}(t+\Delta t)+\boldsymbol{x}(t-\Delta t)-2 \boldsymbol{x}(t)}}{2 \Delta t}=\hat{\mathcal{D}}_{s} \boldsymbol{x},
$$

with

$$
\hat{\mathcal{D}}_{s}=\boldsymbol{u} \cdot \nabla+D \nabla^{2},
$$

and

$$
D=\frac{\overline{(\Delta x)^{2}}}{2 \Delta t}
$$

the diffusion coefficient, assumed to be constant. The symbol $\overline{(\cdot)}$ denotes the aforementioned ensemble averaging.

By considering the forward and backward Fokker-Planck equations for the probability density in $\boldsymbol{x}$-space $\rho(\boldsymbol{x}, t)$ [see, e.g., [18]], and combining them appropriately, it follows that $\rho(\boldsymbol{x}, t)$ is related to the flux velocity through the continuity equation

$$
\frac{\partial \rho}{\partial t}+\nabla \cdot(\rho \boldsymbol{v})=0
$$

and to the diffusive velocity according to

$$
\boldsymbol{u}(\boldsymbol{x}, t)=D \frac{\nabla \rho}{\rho} .
$$

This most important relation confirms the diffusive meaning of the velocity $\boldsymbol{u}$.

The two time derivatives (2) and (4), applied to the velocities (1) and (3), give rise to four different accelerations, thus leading to a couple of generic dynamical equations, which are, respectively, the time-reversal invariant generalization of Newton's Second Law, and the time-reversal non-invariant equation, namely

$$
\begin{aligned}
m\left(\hat{\mathcal{D}}_{c} \boldsymbol{v}-\lambda \hat{\mathcal{D}}_{s} \boldsymbol{u}\right) & =\boldsymbol{f}_{+}, \\
m\left(\hat{\mathcal{D}}_{c} \boldsymbol{u}+\hat{\mathcal{D}}_{s} \boldsymbol{v}\right) & =\boldsymbol{f}_{-},
\end{aligned}
$$

where $\lambda$ is a free, real parameter, and the net force acting on the particle $\boldsymbol{f}$ decomposes as $\boldsymbol{f}=\boldsymbol{f}_{+}+\boldsymbol{f}_{-}$, such that $\boldsymbol{f}_{-}$and $\boldsymbol{f}_{+}$do and do not change sign, respectively, under time reversal (notice that $\boldsymbol{v}$ changes its sign whereas $\boldsymbol{u}$ remains invariant).

Since Equations (8) hold simultaneously and together they describe the dynamics of the system, it is convenient to combine them into a single equation. This is readily achieved by introducing the symbol $\kappa=\sqrt{-\lambda}$ and multiplying the second equation by $\kappa$; the result is

$$
\hat{\mathcal{D}}_{\kappa} \boldsymbol{p}_{\kappa}=\boldsymbol{f}_{\kappa}
$$

with

$$
\begin{aligned}
& \boldsymbol{p}_{\kappa}=m \boldsymbol{w}_{\kappa}+\frac{e}{c} \boldsymbol{A}, \quad \boldsymbol{w}_{\kappa}=\boldsymbol{v}+\kappa \boldsymbol{u}, \\
& \boldsymbol{f}_{\kappa}=\boldsymbol{f}_{+}+\kappa \boldsymbol{f}_{-},
\end{aligned}
$$


and

$$
\hat{\mathcal{D}}_{\kappa}=\hat{\mathcal{D}}_{c}+\kappa \hat{\mathcal{D}}_{s}=\frac{\partial}{\partial t}+\frac{1}{m} \boldsymbol{p}_{\kappa} \cdot \nabla+\kappa D \nabla^{2} .
$$

Equation (9) is the equation of motion appropriate for the description of an ensemble of electrically charged particles immersed in an external electromagnetic field $A$, and subject to stochastic forces. The Newtonian limit (or equivalently, the classical Hamiltonian description) corresponds to $D=0$ and hence $\boldsymbol{u}=\mathbf{0}$, which means no diffusion at all.

For simplicity in the derivations we shall assume no external electromagnetic field $\boldsymbol{A}$, so that the momentum is simply $\boldsymbol{p}_{\kappa}=$ $m \boldsymbol{w}_{\kappa}$ and the external force components reduce to

$$
f_{+}=f=-\nabla V, f_{-}=0 .
$$

Equations (9)-(12) show that the specific dynamical properties of the system strongly depend on the sign of the parameter $\lambda$, which in its turn determines whether $\kappa$ is real or imaginary. Since only the sign of $\lambda$ is relevant [its magnitude can be absorbed into the value of $D$, as explained in de la Peña et al. and Cetto [17, 19]] one can take $\lambda= \pm 1$. The value $\lambda=-1(\kappa=1)$ implies an irreversible dynamics, of the Brownian-motion type. In contrast, by setting $\lambda=1(\kappa=-i)$ one obtains after some algebra the Schrödinger-like equation

$$
-2 m D^{2} \nabla^{2} \psi(\boldsymbol{x}, t)+V(\boldsymbol{x}) \psi(\boldsymbol{x}, t)=2 i m D \frac{\partial \psi(\boldsymbol{x}, t)}{\partial t},
$$

and its complex conjugate, where $\psi(x, t)$ is a complex function such that

$$
\rho(x, t)=|\psi(x, t)|^{2}
$$

and

$$
\boldsymbol{v}=i D\left(\frac{\nabla \psi^{*}}{\psi^{*}}-\frac{\nabla \psi}{\psi}\right), \boldsymbol{u}=D\left(\frac{\nabla \psi^{*}}{\psi^{*}}+\frac{\nabla \psi}{\psi}\right)
$$

whence

$$
\boldsymbol{w}=\boldsymbol{v}-i \boldsymbol{u}=-2 i D \frac{\nabla \psi}{\psi}
$$

\section{THE UNDERLYING EQUATIONS OF STOCHASTIC ELECTRODYNAMICS}

\subsection{The Generalized Fokker-Planck Equation}

We recall that the equation of motion of SED for a (nonrelativistic) particle of mass $m$ and electric charge $e$ is the Langevin equation, also known in SED as Braffort-Marshall equation $[7,14,15]$,

$$
m \ddot{\boldsymbol{x}}=\boldsymbol{f}(\boldsymbol{x})+m \tau \dddot{\boldsymbol{x}}+e \boldsymbol{E}_{0}(t)
$$

where $\tau=2 e^{2} / 3 m c^{3}$, and $\boldsymbol{f}=-\nabla V$. The (random) electromagnetic ZPF is usually taken in the dipole approximation and is therefore represented by $\boldsymbol{E}_{0}(t)$. With the momentum defined as

$$
p=m \dot{x}
$$

Equation (18) transforms into

$$
\dot{\boldsymbol{p}}=\boldsymbol{f}+m \tau \dddot{\boldsymbol{x}}+e \boldsymbol{E}_{0}(t)
$$

Since the dynamics of the system becomes stochastic due to the ZPF, its evolution can only be described in statistical terms. We therefore follow a standard procedure [see de la Peña et al. [17]; section 4.2] that leads to the following generalized Fokker-Planck equation (GFPE) for the phase-space distribution $Q(x, p, t)$,

$$
\hat{L} Q=\left(\hat{L}_{c}+e^{2} \hat{L}_{r}\right) Q=0,
$$

where

$$
\hat{L}_{c}=\frac{\partial}{\partial t}+\frac{1}{m} \nabla \cdot p+\nabla_{p} \cdot \boldsymbol{f}
$$

and

$$
\hat{L}_{r}=\nabla_{p} \cdot\left(\frac{m \tau}{c^{2}} \dddot{\boldsymbol{x}}-\hat{\mathscr{D}}\right)
$$

The operator $\hat{L}_{c}$ contains the classical (i.e., conservative and nondiffusive) Liouvillian terms, and $\hat{L}_{r}$ the radiative and diffusive terms, the latter being represented by the integro-differential operator $\hat{\mathscr{D}}$. To lowest order in $e^{2}$, this operator takes the form

$$
\hat{\mathscr{D}}=\int_{-\infty}^{t} d t^{\prime} \varphi\left(t-t^{\prime}\right) \nabla_{p^{\prime}}
$$

where

$$
\varphi(t)=\frac{2 \hbar}{3 \pi c^{3}} \int_{0}^{\infty} d \omega \omega^{3} \cos \omega t
$$

denotes the ZPF covariance, and $\boldsymbol{p}^{\prime}=\boldsymbol{p}\left(t^{\prime}\right)$ evolves toward $\boldsymbol{p}(t)$ under the action of $\hat{L}$. Notice that it is through this diffusive term that Planck's constant appears in the description.

\subsection{Evolution Equations in Configuration Space}

From Equation (21) follows the equation of evolution in $\boldsymbol{x}$-space for any dynamical variable $G(\boldsymbol{x}, \boldsymbol{p})$ of interest without explicit time-dependence, by left-multiplying the equation by $G$ and integrating over the momentum space. The local mean value of $G$ is

$$
\langle G\rangle_{x} \equiv \frac{1}{\rho} \int d \boldsymbol{p} G(\boldsymbol{x}, \boldsymbol{p}) Q(\boldsymbol{x}, \boldsymbol{p}, t)
$$

where $\rho=\rho_{x}=\rho(\boldsymbol{x}, t)=\int d \boldsymbol{p} Q(\boldsymbol{x}, \boldsymbol{p}, t)$ stands for the probability density. Here we consider only the results corresponding to $G=1$ and $G=p$. In the first case, a direct integration of Equation (21) over $\boldsymbol{p}$ gives the continuity equation for $\rho$,

$$
\frac{\partial \rho}{\partial t}+\nabla \cdot \boldsymbol{j}=0, \boldsymbol{j}=\rho \boldsymbol{v},
$$

with $\boldsymbol{v}=\boldsymbol{v}(\boldsymbol{x}, t)$ the flux (or current) velocity,

$$
\boldsymbol{v}=\frac{1}{m}\langle p\rangle_{x}
$$


For $G=\boldsymbol{p}$ one gets, using (19) and summing over repeated indices,

$$
\frac{\partial}{\partial t} m \boldsymbol{v} \rho+m^{2} \partial_{j}\left\langle\dot{x}_{j} \dot{\boldsymbol{x}}\right\rangle_{x} \rho-\langle\boldsymbol{f}\rangle_{x} \rho=\boldsymbol{R},
$$

with

$$
\boldsymbol{R}=m \tau\langle\dddot{\boldsymbol{x}}\rangle_{x} \rho-e^{2}\langle\hat{\mathscr{D}}\rangle_{x} \rho
$$

containing the radiative and diffusive terms, which are of the order of $e^{2}$.

As is shown in detail in de la Peña et al. [17] (Chapter 4) and de la Peña et al. [20], the left-hand side of Equation (29) can be transformed into the Schrödinger-like equation

$$
-\frac{2 \eta^{2}}{m} \nabla^{2} \psi(\boldsymbol{x}, t)+V(\boldsymbol{x}) \psi(\boldsymbol{x}, t)=2 i \eta \frac{\partial \psi(\boldsymbol{x}, t)}{\partial t}
$$

with $\psi(\boldsymbol{x}, t)$ a complex function such that

$$
\rho(x, t)=|\psi(x, t)|^{2},
$$

$\eta$ a free (undetermined) parameter, and

$$
\boldsymbol{v}(\boldsymbol{x}, t)=\frac{1}{m} \operatorname{Re}\left(\frac{-2 i \eta \nabla \psi}{\psi}\right)=-\frac{i \eta}{m}\left(\frac{\nabla \psi}{\psi}-\frac{\nabla \psi^{*}}{\psi^{*}}\right) .
$$

It is important to note that neither the left-hand side of (29) nor the resulting Equation (31), contain any element that is explicitly related with the ZPF nor with radiation reaction. In fact it is just through the balance eventually achieved between the average energy lost by radiation reaction and that gained from the ZPF (the two terms deriving from the action of $\hat{L}_{r}$, Equation 23), that the value of the parameter $\eta$ is determined. It is thus found that $[17,20]$

$$
\eta=\hbar / 2 \text {, }
$$

which transforms (31) into the true Schrödinger equation; the term on the right-hand side of Equation (29) represents the radiative corrections. We shall come back to this crucial point in section 5.4 .

\section{CONNECTING SED WITH SQM}

\subsection{Comparing the Dynamical Equations}

To explore the connection between the two theories we start by noticing that (33) relates the flux velocity with the real part of the complex vector $(-i \hbar \nabla \psi) / \psi$, while the corresponding imaginary term, on its part, gives the velocity vector

$$
\begin{aligned}
\boldsymbol{u}(\boldsymbol{x}, t) & =-\frac{1}{m} \operatorname{Im}\left(\frac{-i \hbar \nabla \psi}{\psi}\right) \\
& =\frac{\hbar}{2 m}\left(\frac{\nabla \psi}{\psi}+\frac{\nabla \psi^{*}}{\psi^{*}}\right)=\frac{\hbar}{2 m} \frac{\nabla \rho}{\rho} .
\end{aligned}
$$

These expressions coincide precisely with those obtained for the two velocities of SQM, namely Equation (16), if the diffusion coefficient appearing in these equations is assigned the value

$$
D=\frac{\hbar}{2 m} \text {. }
$$

In SED-as in quantum mechanics $-\boldsymbol{v}$ and $\boldsymbol{u}$ represent local ensemble averages; the SQM expressions (1) and (3) represent averages over the ensemble of particles in the neighborhood of $\boldsymbol{x}$, which is a different way of saying the same.

In terms of these velocities, the full SED Equation (29) reads

$$
\begin{aligned}
& m \frac{\partial v_{i}}{\partial t}-m v_{i}\left(\frac{2 m}{\hbar} \boldsymbol{u} \cdot \boldsymbol{v}+\nabla \cdot \boldsymbol{v}\right) \\
& \quad+m\left(\frac{2 m}{\hbar} \boldsymbol{u} \cdot\left\langle\dot{\boldsymbol{x}} \dot{x}_{i}\right\rangle_{x}+\nabla \cdot\left\langle\dot{\boldsymbol{x}} \dot{x}_{i}\right\rangle_{x}\right)=f_{i}+\frac{1}{\rho} R_{i} .
\end{aligned}
$$

For clarity we introduce the tensor $T_{i j}$, given by the (local) correlation between the $i$-th and $j$-th components of the vector $\dot{x}$,

$$
T_{i j}=-\frac{2 m}{\hbar}\left(\left\langle\dot{x}_{i} \dot{x}_{j}\right\rangle_{x}-v_{i} v_{j}\right)=-\frac{2 m}{\hbar}\left(\left\langle\dot{x}_{i} \dot{x}_{j}\right\rangle_{x}-\left\langle\dot{x}_{i}\right\rangle_{x}\left\langle\dot{x}_{j}\right\rangle_{x}\right) \text {, }
$$

so that Equation (36) takes the form

$$
m\left(\frac{\partial v_{i}}{\partial t}-T_{i j} u_{j}-\frac{\hbar}{2 m} \partial_{j} T_{i j}+v_{j} \partial_{j} v_{i}\right)=f_{i}+\frac{1}{\rho} R_{i} .
$$

Barring the radiative corrections, represented by the last term, this dynamical equation reduces to

$$
m\left(\frac{\partial v_{i}}{\partial t}-T_{i j} u_{j}-\frac{\hbar}{2 m} \partial_{j} T_{i j}+v_{j} \partial_{j} v_{i}\right)=f_{i} .
$$

The SQM dynamical equation (8a), in its turn, reads explicitly

$$
m\left(\frac{\partial v_{i}}{\partial t}+v_{j} \partial_{j} v_{i}-u_{j} \partial_{j} u_{i}-D \partial_{j} \partial_{j} u_{i}\right)=f_{i}
$$

in the absence of an external field, when Equation (13) holds. This coincides with the (non-radiative) dynamical equation of SED, Equation (39), with $T_{i j}$ given by

$$
T_{i j}=\partial_{j} u_{i}
$$

Hence, by inserting (41) into (38) we obtain an extended equation for SQM that includes the radiative contributions represented by $R_{i}$,

$$
m\left(\frac{\partial v_{i}}{\partial t}+v_{j} \partial_{j} v_{i}-u_{j} \partial_{j} u_{i}-D \partial_{j} \partial_{j} u_{i}\right)=f_{i}+\frac{1}{\rho} R_{i} .
$$

On the other hand, the SQM Equation (8b) leads after one integration to the continuity equation, which is equivalent to the SED Equation (27).

In this form the connection between SQM and SED is established. The theories are seen to complement one another: while SQM offers the advantage of naturally incorporating from the beginning the couple of velocities $\boldsymbol{v}$ and $\boldsymbol{u}$ to describe the dynamics due to an (unidentified) stochastic source, SED recognizes the ZPF as the determining ingredient that serves to precise the origin of the (quantum) fluctuations, and introduces Planck's constant into the ultimate quantum description. The specific value of $D$ constitutes a postulate in SQM, since in this theory the nature of the stochastic source remains unidentified. Things change when making the connection of SQM with SED, since in the latter theory the ZPF with energy per mode $\hbar \omega / 2$, is the natural carrier of $\hbar$. 


\subsection{Evidence of Diffusion in Quantum Mechanics}

A significant hint of the direct connection of SED and SQM with quantum mechanics follows by observing that the quantum momentum operator is directly related with the velocity $\boldsymbol{w}_{\kappa}$ for $\kappa=-i$ (Equation 17),

$$
\hat{\boldsymbol{p}} \psi=-i \hbar \nabla \psi=(\boldsymbol{v}-i \boldsymbol{u}) \psi .
$$

This result reveals that both velocities $\boldsymbol{v}$ and $\boldsymbol{u}$ are a natural part of quantum mechanics, even if $v$ is rarely used [see however Ballentine [21], and $\boldsymbol{u}$ remains virtually ignored]. In terms of these velocities, the (quantum) expectation value of the squared momentum reads

$$
\left\langle\hat{\boldsymbol{p}}^{2}\right\rangle=m^{2}\left\langle\boldsymbol{v}^{2}+\boldsymbol{u}^{2}\right\rangle,
$$

and the quantum variance

$$
\sigma_{\hat{\boldsymbol{p}}}^{2}=\left\langle\hat{\boldsymbol{p}}^{2}\right\rangle-\langle\hat{\boldsymbol{p}}\rangle^{2}
$$

is given by

$$
\sigma_{\hat{p}}^{2}=\sigma_{m v}^{2}+\sigma_{m u}^{2},
$$

where the variance of a generic vector $\boldsymbol{b}(\boldsymbol{x}, t)$ is given by $\sigma_{\boldsymbol{b}}^{2}=$ $\left\langle\boldsymbol{b}^{2}\right\rangle-\langle\boldsymbol{b}\rangle^{2}$, with $\langle\cdot\rangle=\int d \boldsymbol{x}(\cdot) \rho(\boldsymbol{x}, t)$.

Since

$$
\sigma_{u}^{2}=\left\langle\boldsymbol{u}^{2}\right\rangle=\int d \boldsymbol{x} \rho(\boldsymbol{x}, t) \boldsymbol{u}^{2}(\boldsymbol{x}, t)>0,
$$

momentum dispersion is unavoidable in quantum mechanicsthe single exception being the free particle in a $p$-eigenstate, in which case the position dispersion is infinite. A well-known manifestation of this is the Heisenberg inequality $\Delta x \Delta p \geq \hbar / 2$.

Another distinctive and persisting manifestation of the diffusive velocity $\boldsymbol{u}$ is the so-called quantum potential,

$$
V_{Q}=-\hbar^{2}\left(\nabla^{2} \sqrt{\rho}\right) / 2 \sqrt{\rho}=-\frac{1}{2}\left(m \boldsymbol{u}^{2}+\hbar \nabla \cdot \boldsymbol{u}\right) .
$$

This energy contribution totally due to fluctuations is of paramount importance in determining much of the quantum behavior; we recall that it plays a central role in Bohm's interpretation of quantum mechanics [22].

Along the present discussion we have met the confluence of both theories, SQM and SED, with quantum mechanics, through the equivalence of their statistical nature as being described by the Schrödinger equation. But there is more, since results such as (43)-(46) furnish convincing evidence that, along with the Schrödinger equation, the whole Hilbert-space formalism is involved in such correspondence.

\section{THE MECHANISM OF THE CLASSICAL-TO-QUANTUM TRANSITION}

\subsection{Radiation and Diffusion}

Let us now pay attention to the radiative contributions, represented by the term $e^{2} \hat{L}_{r} Q$ in the GFPE (21). For this purpose we multiply this equation by any constant of motion $G(\boldsymbol{x}, \boldsymbol{p})=\xi$ and integrate over $\boldsymbol{p}$. The terms associated with the classical Liouvillian, $\hat{L}_{c} \xi$, cancel out automatically, and only the two terms associated with $\hat{L}_{r} \xi$ remain. For equilibrium to be reached, these terms must eventually balance each other. By resorting to Equations (24) and (25), one obtains for the balance condition

$$
-\langle\dddot{\boldsymbol{x}} \cdot \boldsymbol{g}\rangle_{x}=\frac{\hbar}{\pi} \int_{0}^{\infty} d \omega \omega^{3} \int_{-\infty}^{t} d t \cos \omega\left(t-t^{\prime}\right)\left\langle\nabla_{p^{\prime}} \cdot \boldsymbol{g}\right\rangle_{x},
$$

with $\boldsymbol{g}(\boldsymbol{x}, \boldsymbol{p})=\nabla_{p} \xi(\boldsymbol{x}, \boldsymbol{p})$ and $\boldsymbol{p}^{\prime}=\boldsymbol{p}\left(t^{\prime}\right), t^{\prime}<t$.

Although the equality in (49) holds only under equilibrium, each side of it can be analyzed separately for all times. It is clear that the two terms reflect different dynamical properties of the system. Whereas, initially (at $t=-\infty$, when particle and ZPF start to interact and there is no diffusion) the radiation term (lefthand side) obviously dominates over the diffusive one (righthand side), with time the diffusion of the momentum increases due to the action of the ZPF. Thus, while the system starts from a non-equilibrium condition, the two dynamical processes allow it to converge toward a balance regime in which the $\xi$ are indeed constant.

Fundamental to the analysis is the factor $\nabla_{p^{\prime}} \cdot \boldsymbol{g}$, which is at the core of the mechanism of evolution toward the balance regime. This coefficient signals the effects on $\boldsymbol{g}(\boldsymbol{x}, \boldsymbol{p})$, of the diffusion of the particles activated by the ZPF through its direct action on the momentum $\boldsymbol{p}$. In classical mechanics, the quantity $\nabla_{p^{\prime}} \cdot g$ can be expressed in terms of a Poisson bracket involving $g$ at time $t$ and $p$ at time $t^{\prime}$,

$$
\frac{\partial g_{i}}{\partial p_{j}^{\prime}}=\left[x_{j}^{\prime}, g_{i}\right] .
$$

The Poisson bracket represents an abridged description of the Hamiltonian evolution, controlled by the classical Liouvillian $L_{c}$; in this case the dynamics is purely deterministic. By contrast, the dynamics contained in Equation (49) is controlled by the entire Liouvillian, and is therefore deterministic in a statistical sense only. This means that although the motion of each particle follows deterministic rules, the fact that it is acted upon by a stochastic field makes the evolution of the ensemble of particles statistically deterministic, hence not amenable to a purely Hamiltonian description. The conforming (modified) Newton equations of motion are of a nature akin to that discussed in section 2 and reflected in Equation (9), which appropriately incorporates the effects of diffusion. As a consequence, the righthand side of Equation (49) - and with it the entire equationceases to obey Hamiltonian laws as soon as the diffusion enters into force.

In conclusion, although initially the dynamics is controlled by Hamiltonian laws, as the interaction develops diffusion eventually takes control. At this point Equation (49) acquires validity, signaling the passage to classical+ZPF physics in the balance regime. The new laws, which are statistical in nature by virtue of the action of the ZPF, coincide with those of quantum mechanics. This means that the Poisson brackets have been replaced by their corresponding commutator. The presence of $\hbar$ in the commutator provides an important clue-although rarely appreciated if at all in its daily use: it is a direct result of the 
crucial role played by the ZPF in the dynamics, and evinces the transition from initially conventional classical to classical+ZPF physics, and eventually to SED in the balance regime, i.e., to quantum physics. That this qualitative change stems from an underlying physical mechanism of transition mastered by the ZPF, may sound natural to some, radical to others; in fact, it is both. Interestingly, however, a qualitative change due to a transition from an initially classical dynamics into one which is fundamentally quantum in nature, has already been observed in experiments with open photonic systems [23].

\subsection{Two Brands of Stochastic Processes}

In the general approach to SQM as briefly discussed in section 2 [and more extensively in, e.g., $[3,17,19]]$, the description of the dynamics involves the undetermined coefficient $\lambda$ that can take the values +1 or -1 , thereby opening the way to the study of two essentially different dynamics. Indeed, this parameter defines the sign of an acceleration related to the diffusion that is to be either added or subtracted to the drift-related acceleration (as shown in Equation 8a), so that the dynamical laws differ from one another, and from the classical (Newtonian) law, due precisely to the diffusive terms. In the referred works and as discussed above, it is shown that the selection $\lambda=-1$ corresponds to Brownian motion, whereas $\lambda=1$ leads to quantum mechanics (through the Schrödinger equation). The close relationship between SQM and SED shows that, despite their dissimilarities, both stochastic processes share certain laws, such as Equations (9-13).

A natural question that emerges from the previous discussion is, how is it that the transition to quantum mechanics occurs in the SED system but not in the case of Brownian motion, which is the most characteristic classical stochastic process? There are several physical features that distinguish the two stochastic processes, a first obvious one being the scale. Whereas, Brownian systems are normally microscopic or macroscopic in size, the quantum ones are of atomic or subatomic size, and many orders of magnitude more sensitive to the relatively high intensity of the stochastic background-in this case the ZPFwhich induces significant fluctuations on the dynamical variables of the system. This difference in the response is so noticeable that one of the first quantum rules to be established (already during 1927) were the Heisenberg uncertainty relations, which in the present understanding express properties of causal stochastic motions, rather than the familiar "inherent" indeterminism. But of course the most important difference refers to the source of the stochasticity, which in the Brownian system is a white noise, free of any self-correlation, whereas in the quantum case it is, according to our description, an intense colored field (due to its $\omega^{3}$-spectrum) with important spatial and temporal selfcorrelations. In fact, as has been shown in the relevant literature [see de la Peña et al. [17] and references therein], it is the radiation field endowed with these high correlations that can be identified as the source of the (statistical) wavelike behavior of quantum particles.

\subsection{Precising the Ontology of Quantum Mechanics}

The question of whether the dynamics of a system can transit from classical to quantum may result misleading or baffling if taken loosely. A legitimate answer requires that the starting theory contain already the ontological elements proper of quantum mechanics. Now, the miscellany of conceptual problems and difficulties that beset conventional quantum mechanics, when closely looked at, point toward the possibility of a common origin, namely some critical component that has been left aside. Here we are proposing to consider the zero-point radiation field as the key missing element in the quantum ontology, and the transition, therefore, not from plain classical physics but from classical-plus-zero-point-radiationfield physics to quantum physics. As seen from the above analysis, this more complete ontology leads in a natural process to the quantum description.

Equation (31), along with Equations (43-46), imply that once the balance (or quantum) regime is established, the dynamical variables can legitimately be treated via the corresponding usual operators in Hilbert space. This perspective stands in contrast with the historical one, in which the founders of the theory felt compelled to introduce operators (in their matrix representation) to account for the observed facts-just as Newton's law of gravitation was proposed to save the phenomenon-without any acknowledgment (nor knowledge) of the role played by the underlying cause, the theoretical weight of which remained-and still remains-largely unrecognized, adding its part to the opacity of quantum mechanics.

The perspective to be drawn from these results is that the ZPF not only plays a significant role in explaining quantum indeterminism as the result of an induced stochasticity, but that its presence provides the basis for an explanation of the quantum behavior of matter altogether. [A more extensive discussion and substantiation of these matters is presented in de la Peña et al. [17]].

A note about the reverse transition from quantum to classical seems appropriate at this point. It is usual to consider classical physics as a limiting case of the quantum description, attained e.g., by allowing $\hbar$ to go to zero, with the argument that in this limit all operators commute. This is however a formal transition; the fundamental difference in the nature of the classical-vsquantum dynamics demands a more in-depth consideration of this apparently simple "change of scale."

\subsection{Some Words About the Radiative Corrections}

Equation (42) is the dynamical law of both SED and SQM, including the radiative corrections to second order in e. This more complete description allows one to obtain several important results pertaining to the realm of quantum electrodynamics (in the non-relativistic approximation), such as the formulas for the Einstein coefficients, which determine in particular the lifetimes of atomic states. The corresponding calculations and results can be seen in de la Peña et al. [17] and references therein. In the context of the present work, the most interesting application has been the determination of the diffusion coefficient $D$ of SQM.

We recall that according to the SED Equation (49), a balance must be achieved in the quantum regime between the radiative and dissipative effects on the dynamics. In particular, for $\xi=\boldsymbol{p}^{2} / 2 m+V$, Equation (49) represents the energy-balance 
condition, meaning that the mean power absorbed by the particle from the ZPF is compensated by the mean power radiated by the former. While the radiation reaction term contains parameters deriving from (classical) electrodynamics only, Planck's constant enters into the second term through the spectral energy density of the ZPF. A detailed calculation of the two terms shows that it is precisely this balance condition what fixes uniquely the value of the free parameter $\eta$ used in section 3.2, and hence of the diffusion coefficient $D$ in terms of $\hbar$.

\section{FINAL REMARKS AND CONSIDERATIONS}

For several decades already, two theories have coexisted which arrive at quantum mechanics from an (assumed) classical context that includes stochasticity as an essential ingredient. Historically they were developed by different and virtually independent clusters of researchers, with little intersection. Hence their coexistence has been more than peaceful. Also their philosophies are quite distant, SQM having been conceived of as a Browniantype theory for the particle subject to a white noise from an unidentified source. By contrast, SED has been developed as a statistical description for the particle subject to the ZPF with a colored spectrum. As shown here, the two theories complement each other and both lead to the Schrödinger equation after appropriate workings; thus, in the global scenario quantum mechanics emerges from a classical+stochastic context. Leaving aside the theoretical body here developed, one could ask, why so?

The reason for the success of such parallel constructs is traced to the role played by diffusion. In SQM the velocity $\boldsymbol{u}$ is introduced from the very start as a dynamical variable that encapsulates the diffusive effect of the random force on the particle motion. Both the diffusive velocity $\boldsymbol{u}$ and the flux velocity $\boldsymbol{v}$ are of course statistical concepts, and together with the ensuing four accelerations they modify Newton's Second Law in an essential way. Also SED starts by considering the appropriate

\section{REFERENCES}

1. Fényes I. Eine wahrscheinlichkeitstheoretische Begründung und Interpretation der Quantenmechanik. Zeits Physik. (1952) 132:81-106. doi: 10.1007/BF01338578

2. Nelson E. Dynamical Theories of Brownian Motion. Princeton, NJ: Princeton University Press (1967).

3. de la Peña L. New formulation of stochastic theory and quantum mechanics. $J$ Math Phys. (1969) 10:1620. doi: 10.1063/1.1665009

4. Guerra F. Structural aspects of stochastic mechanics and stochastic field theory. Phys Rep. (1981) 77:263-321. doi: 10.1016/0370-1573(81)90 078-8

5. Gaveau B, Jakobson T, Kac M, Schulman LS. Relativistic extension of the analogy between quantum mechanics and Brownian motion. Phys Rev Lett. (1984) 53:419-22. doi: 10.1103/PhysRevLett. 53.419

6. Nelson E. Quantum Fluctuations. Princeton, NJ: Princeton University Press (1985).

7. de la Peña L, Cetto AM. The Quantum Dice. Dordrecht: Kluwer Academic Publishers (1996).

8. Nelson E. Review of stochastic mechanics. JPCS. (2012) 361:012011. doi: 10.1088/1742-6596/361/1/012011 statistical description by means of the GFPE, which ensues from the (stochastic) Langevin-type equation-equally modifying the Second Law in an essential way.

In this work we have established the equivalence between the equations of motion derived in SED-a fundamental theoryand those of $S Q M-$ a phenomenological theory. One may say that SQM becomes thus explained by SED, and completed by it. This is reinforced by recalling that the value of the diffusion constant $D=\hbar / 2 m$-a free postulate in SQM, which has no natural place for Planck's constant-is derived from a consideration of the radiative terms of SED, as explained in section 5.4 .

A final important point is that the coherence between the SQM and SED theories unravels in the former the presence of an undulatory element, which it lacks of in its usual strictly corpuscular treatments [by Nelson and followers; see Nelson and Guerra [2, 4]]. This provides a natural answer to the wellknown objection against SQM by Wallstrom [24, 25], who deems the known derivations incorrect, based on the argument that they require an ad hoc (wave-like) quantization condition on the velocity potential (the gradient of which gives the velocity $\boldsymbol{v}$ ) in order to derive Schrödinger's equation; of course such condition appears artificial in a strictly corpuscular framework, but it acquires a natural place in a theory that embodies a radiation field as its substantial source of stochasticity.

\section{AUTHOR CONTRIBUTIONS}

All authors listed have made a substantial, direct and intellectual contribution to the work, and approved it for publication.

\section{ACKNOWLEDGMENTS}

Useful comments from the referees are acknowledged. The authors acknowledge financial support from DGAPA.UNAM through project PAPIIT IN113720.
9. Khrennikov AY. Beyond Quantum. Taylor \& Francis; Boca Raton, FL: CRC Press (2014).

10. t'Hooft G. The Cellular Automaton Interpretation of Quantum Mechanics. Berlin: Springer Verlag (2016).

11. Marshall TW. Random electrodynamics. Proc R Soc A. (1963) 276:475. doi: $10.1098 /$ rspa.1963.0220

12. Marshall TW. Statistical electrodynamics. Proc Camb Philos Soc. (1965) 61:537.

13. Boyer TH. Random electrodynamics: the theory of classical electrodynamics with classical electromagnetic zero-point radiation. Phys Rev D. (1975) 11:790-808. doi: 10.1103/PhysRevD.11.790

14. Claverie P. Stochastic electrodynamics versus quantum theory: recent advances in the study of non-linear systems. In: Moore SM, et al., editors. Proceedings of Einstein Centennial Symposium on Fundamental Physics. Bogotá: Universidad de los Andes (1981). p. 229.

15. Santos E. Locality in stochastic electrodynamics. In: Moore SM, et al., editors. Proceedings of Einstein Centennial Symposium on Fundamental Physics. Bogotá: Universidad de los Andes (1981). p. 213.

16. de la Peña L, Cetto AM. Derivation of quantum mechanics from stochastic electrodynamics. J Math Phys. (1977) 18:1612. doi: 10.1063/1.523448

17. de la Peña L, Cetto AM, Valdés-Hernández A. The Emerging Quantum. Berlin: Springer Verlag (2015). 
18. Risken H. The Fokker-Planck Equation. Methods of Solution and Applications. Berlin: Springer Verlag (1984).

19. Cetto AM, de la Peña L, Valdés-Hernández A. Specificity of the Schrödinger equation. Quant Stud Math Found. (2015) 2:275-87. doi: 10.1007/s40509-015-0047-5

20. de la Peña L, Cetto AM, Valdés-Hernández A. The zero-point field and the emergence of the quantum. Int J Mod Phys E. (2014) 23:1450049. doi: 10.1142/S0218301314500499

21. Ballentine LE. Quantum Mechanics: A Modern Development. Singapore: World Scientific (1998).

22. Bohm D, Hiley BJ. The Undivided Universe. New York, NY: Routledge (1995).

23. Raftery J, Sadri D, Schmidt S, Türeci HE, Houck AA. Observation of a dissipation-induced quantum transition. Phys Rev X. (2014) 4:031043. doi: 10.1103/PhysRevX.4.031043

24. Wallstrom TC. On the derivation of the Schrödinger equation from stochastic mechanics. Found Phys Lett. (1989) 2:113.
25. Wallstrom TC. Inequivalence between the Schrödinger equation and the Madelung hydrodynamic equation. Phys Rev A. (1994) 49:1613. doi: 10.1103/PhysRevA. 49.1613

Conflict of Interest: The authors declare that the research was conducted in the absence of any commercial or financial relationships that could be construed as a potential conflict of interest.

Copyright (c) 2020 de la Peña, Cetto and Valdés-Hernández. This is an open-access article distributed under the terms of the Creative Commons Attribution License (CC $B Y)$. The use, distribution or reproduction in other forums is permitted, provided the original author(s) and the copyright owner(s) are credited and that the original publication in this journal is cited, in accordance with accepted academic practice. No use, distribution or reproduction is permitted which does not comply with these terms. 